\title{
Fingerprinting with MRI
}

A new approach to magnetic resonance imaging data acquisition and analysis promises to improve sensitivity and facilitate quantitative measurements.

Despite the wide use of magnetic resonance techniques in basic research and in the clinic, methodological improvements that sharpen these tools are always useful.

Mark Griswold and his team at Case Western Reserve University have been pursuing ways to improve magnetic resonance imaging (MRI) technology, in particular for clinical translation. "One of the biggest problems in MRI has been the fact that it's completely qualitative in most cases," he notes. "Most of the time when we look at an image, we see that it's bright on one side and dark

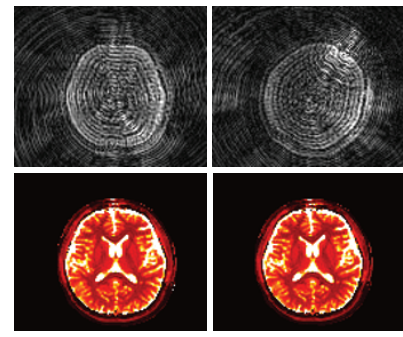

Magnetic resonance fingerprinting maps (bottom) are tolerant to errors caused by a human subject moving his head (top). Reprinted from Nature. on another, and that tells us something about the disease that's there, but it's always a relative measure." Past attempts to solve this problem have substantially increased data acquisition time or lowered measurement sensitivity and have therefore not been practical for use in a patient.

Griswold's team's new approach to MRI data acquisition and analysis promises to change this. Their method, dubbed 'magnetic resonance fingerprinting', or MRF, provides a conceptually new way of acquiring and processing magnetic resonance data.

In an MRI scan, magnetic resonance parameters-which can include longitudinal $\left(T_{1}\right)$ and transverse $\left(T_{2}\right)$ relaxation times, off-resonance frequency, spin density, diffusion and magnetization transfer-are serially collected. These parameters reflect the microenvironment of proton spins in water molecules in a tissue or material. The images created are qualitative, with the contrast typically weighted to just one type of parameter.

\section{GENETICS}

\section{CRISPR SILENCING}

An enzymatically dead Cas 9 endonuclease permits targeted, RNA-guided transcriptional silencing.

Reverse genetics has never been quite the same since the development of RNAi. The ability to knock down just about any gene and to do so at a large scale-hundreds or thousands of genes or more-forever changed the way in which the genotype-phenotype relationship could be probed in many organisms. Nevertheless, RNAi does have its drawbacks: it does not always work effectively, and off-target effects can be a real problem.

Lei Qi, a Systems Biology Fellow at the University of California, San Francisco (UCSF), working in collaboration with the laboratories of Wendell Lim and Jonathan Weissman, also at UCSF, and of Jennifer Doudna and Adam Arkin at UC Berkeley, sought to develop an approach for genetic perturbation that combines the simplicity and scalability of RNAi with the specificity of methods based on targeted DNA-binding proteins (that is, the zinc-finger proteins and the transcription activator-like effectors).

The researchers settled on the clustered, regularly interspaced, short palindromic repeats (CRISPR) system, which is part of the protective response of bacteria and archaea to foreign DNA. In this system, external DNA is sequence-specifically cleaved by the combined action of protein and RNA components, a process that has recently been harnessed to effect targeted genome editing in several organisms. In one of the simplest versions of CRISPR, that from the bacterium Streptococcus pyogenes, target recognition and cleavage can be achieved with a single protein, the CRISPR-associated endonuclease Cas9, and a single engineered guide RNA (gRNA).

But there was one glaring problem with CRISPR, explains Qi. “0ne barrier to what we wanted to achieve is that Cas 9 is an endonuclease. Since we wanted to regulate gene expression reversibly and transiently and without changing the genotype, we didn't want the nuclease activity." The researchers therefore engineered into the endonuclease mutations known to render it enzymatically dead. The dead Cas9, they saw, could still bind gRNA and then the cognate DNA target. 
Instead the serial scanning for various parameters, in MRF the parameters are continuously varied during data acquisition such that the signals arising from the sample constitute a unique 'fingerprint'. The heart of the method is a pattern recognition algorithm that matches these fingerprints to a dictionary of predicted signal patterns for the tissue or material under investigation. One needs to know only the range of signal patterns to expect to predict the dictionary entries, notes Griswold. "In the human body, we know the range of $T_{1} \mathrm{~s}$, we know the range of $T_{2}$ s, we know the range of off-resonances [and so on]," he explains.

In this way, MRF allows the researchers to assign hard numbers to the signals they detect rather than just generating qualitative maps. Such a feature should be useful not only in the clinic but for all research using magnetic resonance techniques, including nuclear magnetic resonance spectroscopy. The method also provides substantially higher sensitivity and precision than any previous method. "That and always being able to put quantitative numbers on it should really change the way we do things," says Griswold.

Because MRF is based on pattern recognition, it is much more tolerant than conventional MRI to measurement errors, such as those arising when a patient getting a brain scan moves his or her head slightly. MRF could therefore allow clinicians and researchers to obtain higherquality results from current-generation scanners or even to obtain satisfactory results using cheaper scanners.

An interesting potential application of MRF is the detection of early cancerous cells in tissue. "We know that cancer cells are different at the molecular level; they're different at the microscopic level," explains Griswold. Such differences would be reflected as unique cancer cell fingerprints by MRF, potentially helping to improve diagnoses. Although his lab is largely focused on medical applications, he is also working with collaborators in basic research fields to implement MRF. "Hopefully we can get higher and higher sensitivity across the board if we do this right," he says.

\section{Allison Doerr}

\section{RESEARCH PAPERS}

Ma, D. et al. Magnetic resonance fingerprinting. Nature 495, 187-192 (2013).

Would the Cas9 complex perturb transcription when it bound DNA in the genomic context? From the way in which transcriptional repressors such as LacI are thought to function (essentially as roadblocks to the transcribing RNA polymerase), Qi and colleagues thought that it might.

Indeed, the researchers showed that the modified CRISPR system can substantially reduce the levels of an integrated red fluorescent protein reporter in Escherichia coli. The silencing effect occurs at the level of transcription and requires targeting of the gRNA to the nontemplate strand. As determined by RNA-seq, the only transcript that was significantly knocked down in these bacteria was that encoding red fluorescent protein, indicating that the approach is specific. Qi and colleagues went on to show that the system is reversible, can be used to target multiple genes simultaneously and can achieve knockdown by a factor of up to 1,000 with the design of multiple nonoverlapping gRNAs targeting the same gene. They also showed that the CRISPR system is effective on endogenous genes of the lac operon.

The knockdown efficiency depends on both the gRNA position on the target gene and the degree of mismatch between gRNA and its target sequence. The researchers expect that a systematic study of these effects, ongoing in their laboratories, will yield a system that is tunable in a predictable way so that something akin to an allelic series of mutations can be generated using this approach, which they named CRISPRi.

In human cells, the efficiency of CRISPRi does not at present approach that in $E$. coli. But Qi is optimistic that further work will render the approach useful in human cells and other eukaryotic systems.

The researchers also hope to use the enzymatically dead Cas9 as a way to target other activities-chromatin modification, for example-to specific locations in the genome. The possibilities of a targeted genome-perturbation method that is also simple, specific, flexible and dynamic seem almost endless.

\section{Natalie de Souza}

